\title{
The circle of Willis predicts the antihypertensive effects of carotid artery stenting
}

\author{
*David R. Santiago-Dieppa, MD, Brian R. Hirshman, MD, PhD, Arvin Wali, BA, \\ J. Scott Pannell, MD, Yasaman Alam, BS, Scott Olson, MD, Vincent J. Cheung, MD, \\ Jeffrey A. Steinberg, MD, Mihir Gupta, MD, and Alexander A. Khalessi, MD, MS \\ Department of Neurosurgery, University of California, San Diego, California
}

\begin{abstract}
OBJECTIVE Carotid artery stenting (CAS) has antihypertensive effects, but the durability and degree of this response remain variable. The authors propose that this clinical variability is a function of the presence or absence of a complete circle of Willis (COW). Incomplete COWs perfuse through a higher-resistance pial collateral pathway, and therefore patients may require a higher mean arterial pressure (MAP). Carotid artery revascularization in these patients would reduce the end-organ collateral demand that has been hypothesized to drive the MAP response.
\end{abstract}

METHODS Using a retrospective, nonrandomized within-subject case-control design, the authors compared the postoperative effects of CAS in patients with and without a complete COW by using changes in MAP and antihypertensive medication as end points. They recorded MAP and antihypertensive medications 3 months prior to surgery, preoperatively, immediately postoperatively, and at the 3-month follow-up.

RESULTS Data were collected from 64 consecutive patients undergoing CAS. Patients without a complete COW (25\%) were more likely to demonstrate a decrease in BP response to stenting (i.e., a drop in MAP of $10 \mathrm{~mm} \mathrm{Hg}$ and/or a reduction or cessation of BP medications at 3 months postoperatively). Of the patients in the incomplete COW cohort, $75 \%$ had this outcome, whereas of those in the complete COW cohort, only $41 \%$ had it $(p<0.041)$. These findings remained statistically significant in a logistic regression analysis for possible confounders $(p<0.024)$. A receiver operating curve analysis of preoperative data indicated that a MAP $>96.3 \mathrm{~mm} \mathrm{Hg}$ was $55.5 \%$ sensitive and $57.4 \%$ specific for predicting a complete COW and that patients with a MAP $>96.3 \mathrm{~mm} \mathrm{Hg}$ were more likely to demonstrate a good MAP decrease following CAS $(p<0.0092)$.

CONCLUSIONS CAS is associated with a significant decrease in MAP and/or a reduction/cessation in BP medications in patients in whom a complete COW is absent.

https://thejns.org/doi/abs/10.3171/2017.1.FOCUS16487

KEY WORDS carotid artery stenosis; hypertension; blood pressure; angioplasty and stenting; carotid artery stent; pial collaterals; circle of Willis

$\mathrm{T}$ HE diagnosis and treatment of hypertension and atherosclerotic stenosis of the carotid artery are imperative clinical objectives for preventing stroke, $, 8,14$ and yet the pathophysiological mechanisms linking these two diseases remains incompletely characterized. In addition to the transient carotid artery baroreceptor-medi- ated hypotension that may complicate carotid artery stent (CAS) placement, ${ }^{17}$ stenting has been shown to exert durable reductions in postoperative blood pressure (BP) and requirements for antihypertensive medications. ${ }^{1,6,7}$ However, the degree of hypertensive reduction and the durability of this effect after CAS remain highly variable, with some

ABBREVIATIONS ACOA = anterior communicating artery; $\mathrm{BP}=$ blood pressure; $\mathrm{CAS}=$ carotid artery stent; $\mathrm{COW}=$ circle of Willis; $\mathrm{MAP}=$ mean arterial $\mathrm{BP} ; \mathrm{PCOA}=$ posterior communicating artery; $\mathrm{ROC}=$ receiver operating characteristic; $\mathrm{TIA}=$ transient ischemic attack.

SUBMITTED November 15, 2016. ACCEPTED January 25, 2017.

INCLUDE WHEN CITING DOI: 10.3171/2017.1.FOCUS16487.

* Drs. Santiago-Dieppa and Hirshman contributed equally to this work. 
studies reporting no significant long-term changes in $\mathrm{BP}$ after stent placement. ${ }^{13}$

Intracranial collateral flow is critical for facilitating compensatory perfusion during periods of cerebral ischemia and, anatomically, ranges from the larger-diameter circle of Willis (COW) to the tiny-caliber pial (leptomeningeal) collaterals (Fig. 1). ${ }^{16}$ Recently, pial collateral pathways of perfusion have been increasingly recognized as an important mechanism of adaptation for cerebral ischemia, and they prognosticate a response to therapeutic intervention and recovery from acute stroke. ${ }^{2,5,10-12}$ The absence of a complete COW necessarily drives perfusion across a smaller-diameter and higher-resistance pial collateral gradient. We postulate that this greater dependence on a high-resistance collateral pathway relies on a higher pretreatment mean arterial BP (MAP) and thereby forms the anatomical and physiological basis for the highly variable BP reduction reported after CAS. Based on this hypothesis, patients with an incomplete COW would have a greater reduction in the driving force of perfusion (i.e., MAP) relative to patients with a complete COW following CAS.

\section{Methods}

This retrospective cohort study, which met all institutional oversight and regulations and did not place patients at any additional risk of harm, received IRB approval. Patients were included if they had a carotid artery stent placed between November 2013 and October 2016 for symptomatic carotid artery stenosis (i.e., transient ischemic attack [TIA] or prior stroke) or as indicated by current guidelines. ${ }^{3,8}$ Patients were excluded from this study if they did not meet the aforementioned criteria or were younger than 18 years of age. Seventy-four consecutive patients met the inclusion criteria, of whom 10 were lost to follow-up, ultimately leaving 64 in the final analysis cohort. A subset of patients $(n=42)$ had been previously seen by primary care or subspecialty care 3 months prior to surgery, allowing that subset of patients to serve as their own controls. As such, this project was conceived as a nonrandomized, within-subject design that allowed for control of personal and lifestyle factors not otherwise affected by CAS.

\section{Cohort Definitions and Description of Outcome Measures}

Sixteen of the 64 patients (25\%) had either an absent anterior communicating artery (ACoA) or ipsilateral posterior communicating artery $(\mathrm{PCoA})$ found on review of intraoperative angiograms and/or available pretreatment vascular images. These 16 patients were considered part of the "pial collateral-dependent circulation" cohort (i.e., they had an incomplete COW), while the remainder of the patients $(\mathrm{n}=48)$ who had both vessels (i.e., they had a complete COW) were considered the "complete collateral circulation" cohort. Examination of patients and data collection took place at 4 points in time: 3 months before surgery, immediately preoperatively, immediately postoperatively, and 3 months after surgery. The primary end point of comparison between the cohorts in the study was whether a meaningful BP response to stenting was achieved through comparison of BP 3 months after stent-
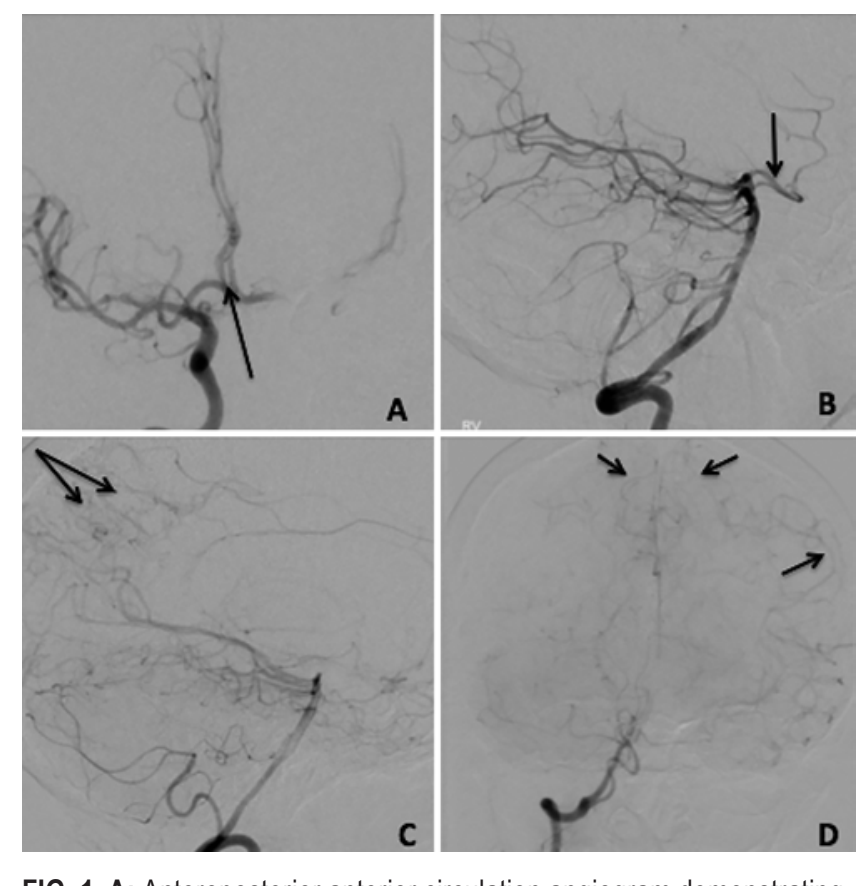

FIG. 1. A: Anteroposterior anterior circulation angiogram demonstrating a complete $\mathrm{COW}$ with a patent ACoA. B: Lateral posterior circulation angiogram demonstrating complete $\mathrm{COW}$ and patent PCoAs. C: Lateral posterior circulation angiogram in a patient with incomplete COW in the setting of carotid artery stenosis, demonstrating retrograde pial or leptomeningeal collateralization of the middle cerebral artery (MCA) and anterior cerebral artery territory. D: Anteroposterior right vertebral artery angiogram in a patient with near-complete left internal carotid artery stenosis and incomplete COW, demonstrating pial collateral backfilling of bilateral $A_{1}$ segments and MCA distribution. Arrows identify pial collaterals.

ing with BP immediately before stenting. We defined a meaningful response as a drop in MAP of at least $10 \mathrm{~mm}$ $\mathrm{Hg}$ and/or a decrease or cessation in one or more BP medications. We also examined whether a similar meaningful change, again defined as a drop in MAP of at least $10 \mathrm{~mm}$ $\mathrm{Hg}$ and/or a decrease or cessation in one or more BP medications, occurred in the 3 months prior to intervention.

\section{Statistical Analysis}

R software version 3.3.1 (http://www.r-project.org) was used for the descriptive statistical analysis. The categorical variables were compared using the Fisher exact test and a multivariable binary logistic regression analysis. Variables included in the multivariable model were presence of complete COW, age, sex, history of TIA or stroke, increase in BP medications after surgery, presence of hypertension, hyperlipidemia, and diabetes mellitus. Sensitivity analysis with different decreases in MAP was also conducted. A receiver operating characteristic (ROC) curve was used to define the cutoff MAP, sensitivity, and specificity of predicting which patients lacked a complete COW. For all data analysis, $\mathrm{p}<0.05$ was considered statistically significant.

\section{Results \\ Data Set}

Rates of comorbid conditions did not vary across the 


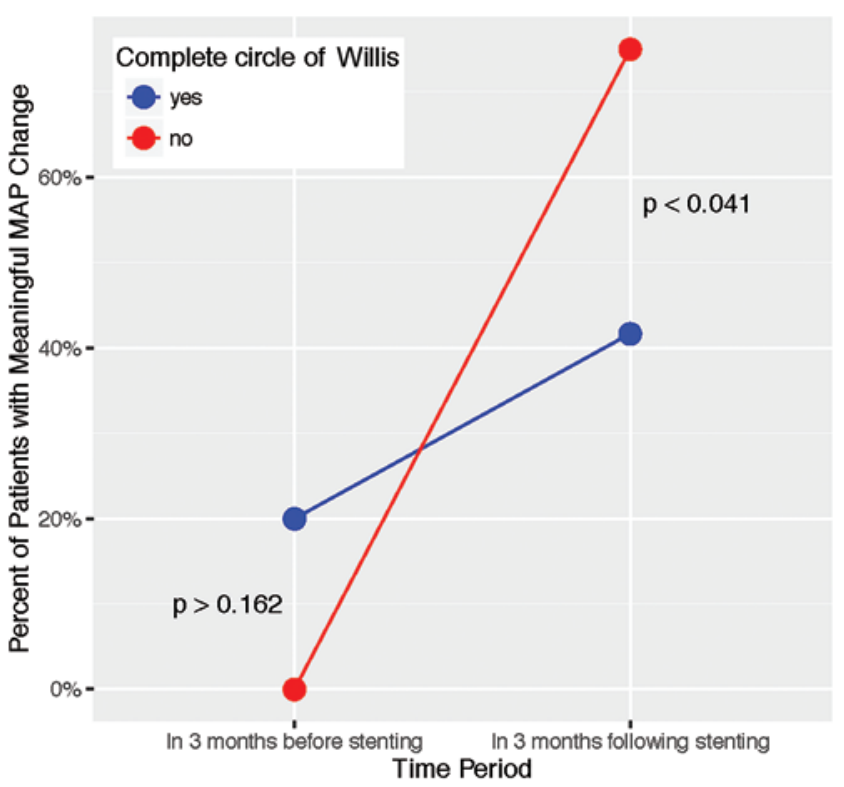

FIG. 2. After stratifying patients by the presence (blue) or absence (red) of a complete ipsilateral COW, BP and antihypertensive medications were assessed in the 3 months before unilateral CAS (left time point) or the 3 months following unilateral CAS (right time point). The $y$-axis illustrates the proportion of patients having a meaningful drop in BP, defined as either a decrease in MAP by $10 \mathrm{~mm} \mathrm{Hg}$ or a decrease in BP medication numbers and dosing. Following CAS, patients dependent on high-resistance pial collaterals had a statistically significant and durable reduction in hypertension $(p<0.041)$ compared with patients with a complete COW. This difference was not present prior to stenting ( $p>0.162)$.

pial circulation cohort and the collateral circulation cohort. Across both cohorts, patients were $70.3 \%$ male, and $46.8 \%$ were older than 70 years of age. A total of 45 patients $(70.3 \%)$ had had a prior stroke or TIA that had resolved by the time of carotid artery stent placement. The majority of patients had a prior diagnosis of hypertension (76.5\%), diabetes (28.1\%), and/or hyperlipidemia $(60.3 \%)$.

\section{Patients Pial Collateral-Dependent Circulation Have a Greater BP Response to Stenting}

Patients in the pial collateral cohort were more likely to demonstrate a good BP response to stenting (defined as a drop in MAP of $10 \mathrm{~mm} \mathrm{Hg}$ or decrease in/cessation of one or more BP medications at 3 months). Twelve (75\%) of the 16 of the patients in the pial collateral cohort had this outcome, while only $20(41.6 \%)$ of the 48 patients in the collateral circulation cohort did so ( $p<0.041$ by Fisher exact testing, Fig. 2). By comparison, only 6 patients had a meaningful drop in BP prior to intervention-and all patients were in the collateral circulation cohort $(\mathrm{p}>$ 0.162 , Fisher exact test). Sensitivity analysis with different decreases in MAP did not appreciably change the outcome.

When adjusting for potential confounders via multivariable logistic regression, including hypertension, increases in BP medications after surgery, and hyperlipidemia, the pial collateralization cohort remained an independent pre- dictor for a meaningful drop in BP 3 months after stenting (6.014 odds ratio, $\mathrm{p}<0.024)$.

The drop in MAP after intervention was entirely a long-term response to stenting, as this decrease in BP was not observed at the immediate postoperative time point ( $\mathrm{p}$ $>0.41$ ). Furthermore, there was no immediate or sustained decrease in systolic BP. Using an analogous definition of meaningful drop in BP (a drop in systolic BP of $10 \mathrm{~mm}$ $\mathrm{Hg}$ or a decrease in/cessation of one or more BP medications at 3 months), only $10(62.5 \%)$ of the 16 patients in the pial collateral cohort demonstrated such a drop, versus 27 $(54 \%)$ of 46 in the collateral circulation cohort ( $>0.772$ ).

\section{MAP > $96.3 \mathrm{~mm} \mathrm{Hg}$ Predicts Dependence on Pial Collaterals}

There was an appreciable difference in the immediate presurgical MAP between the pial collateral cohort and collateral circulation cohort. The median MAP was 97 $\mathrm{mm} \mathrm{Hg}$ in the pial collateralization cohort (range 69-113 $\mathrm{mm} \mathrm{Hg}$ ) versus $94 \mathrm{~mm} \mathrm{Hg}$ in the collateral circulation cohort (range $69-130 \mathrm{~mm} \mathrm{Hg}$ ). A ROC analysis of preoperative data indicated that MAP > $96.3 \mathrm{~mm} \mathrm{Hg}$ was $55.5 \%$ sensitive and $57.4 \%$ specific for predicting patients who lacked collateral circulation from either the ACoA or ipsilateral PCoA (i.e., that such patients were members of the pial collateral cohort) and that patients with MAPs above $96.3 \mathrm{~mm} \mathrm{Hg}$ were more likely to demonstrate a MAP decrease following CAS ( $\mathrm{p}<0.0092)$.

\section{Discussion}

Hypertension and carotid artery stenosis secondary to atherosclerosis remain widely prevalent diseases that commonly coexist. ${ }^{4,14,15}$ While the pathogenesis of these two diseases is known to intersect, the pathophysiological mechanism that these diseases share is not well established. Several studies have examined the clinical response that treating carotid artery stenosis had on BP, with most finding a greater reduction of hypertension in stenting group compared with the carotid endarterectomy group. ${ }^{8}$ Thus, while an association between hypertension and carotid artery stenosis exists, there are currently no mechanistic or clinical investigations to guide patient selection for carotid artery revascularization based on the presence of hypertension nor to predict the long-term therapeutic response associated with CAS.

In the present study, we found that CAS resulted in a statistically significant reduction in MAP and/or a decrease in BP medications at the 3-month time point for patients who were completely dependent on pial collateral pathways. This good BP response to stenting was observed in $75 \%$ of the patients in the pial collateral cohort, compared with only $41 \%$ of the COW collateral circulation cohort $(\mathrm{p}<0.041)$. Additionally, we observed that there was an appreciable difference in MAP between the cohorts. To further characterize this observation, we performed a secondary ROC analysis of preoperative data, which indicated the following: 1) MAP > $96.3 \mathrm{~mm}$ $\mathrm{Hg}$ was $55.5 \%$ sensitive and $57.4 \%$ specific for predicting which patients lacked a complete COW, and 2) patients with a MAP > $96.3 \mathrm{~mm} \mathrm{Hg}$ were more likely to 
Final Patient Cohort $(\mathrm{n}=64)$ with Symptomatic Carotid Disease

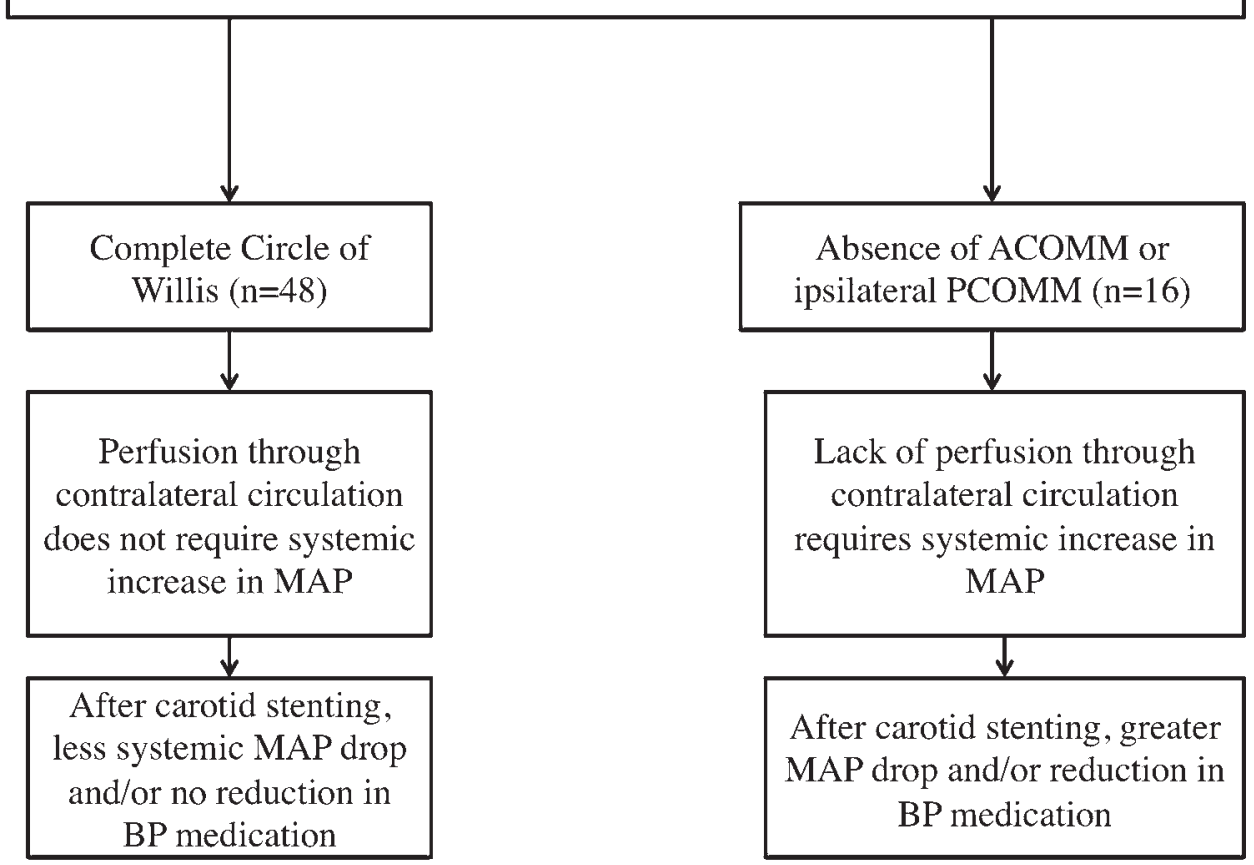

FIG. 3. Distribution of patients between complete COW cohort $(n=48)$ and pial collateral cohort $(n=16)$. ACOMM = ACoA; $\mathrm{PCOMM}=\mathrm{PCOA}$.

demonstrate a statistically significant decrease in MAP following stenting $(\mathrm{p}<0.0092)$. Moreover, these findings remained statistically significant $(\mathrm{p}<0.024)$ when adjusting for possible confounders such as systemic hypertension, increases in BP medications, and hyperlipidemia.

Importantly, our findings exclusively represent a longterm response to stenting, as relative differences in MAP reduction between the two cohorts were not observed during the immediate postoperative period. These findings offer new insights into a potential mechanism of BP reduction that is unique from the response mediated by the carotid sinus. ${ }^{9}$ Poiseuille's law of fluid dynamics demands that to maintain constant cerebral blood flow in individuals lacking a complete COW, perfusion must occur through an alternative, smaller-caliber and higher-resistance pathway. This proposed anatomical mechanism offers a new explanation as to why carotid revascularization in these patients would reduce the end-organ collateral demand hypothesized to drive the higher MAP response, and it further emphasizes the importance of preoperative cranial imaging to assess collateral pathways.

Our study is among the first to suggest that chronic intracranial hypoperfusion may be a cause of secondary hypertension. Numerous studies, and indeed many of our current stroke guidelines, have previously emphasized that BP drives end-organ perfusion in the acute setting., ${ }^{4,14,15}$ Our study suggests that inadequate intracranial perfusion via high-resistance pial pathways may be an additional factor that increases long-term systemic BP and that these effects are independent of confounders such as diabetes and hyperlipidemia. While secondary causes of hypertension such as renal artery stenosis, excessive fluid or volume status, pheochromocytoma, hypercortisolism, hyperaldosteronism, and lifestyle factors have been previously described, this is, to the best of our knowledge, the first study to specifically explore the interaction of intracranial and extracranial anatomy as a cause of sustained systemic hypertension. If hypertension is driven in part by intracranial demand, as our results suggest, further screening and intervention may be helpful in the general population of patients with resistant hypertension.

Despite the inherent retrospective limitations of this small study with only 3 months of follow-up, it offers new insights into a previously undescribed population of patients with coexistent hypertension and symptomatic carotid artery stenosis who appear to respond more robustly to carotid revascularization. The findings of this study may have implications for expanding the definition of "symptomatic" cervical carotid artery stenosis to eventually include hypertension, and they suggest that characterization of the COW may be beneficial in predicting a therapeutic reduction in BP after carotid revascularization (Figs. 3 and 4). Furthermore, a MAP $>96.3 \mathrm{~mm} \mathrm{Hg}$ may be a potential threshold for this expanded definition. Ultimately, the next step in further characterizing this newly described population of patients is a larger prospective trial.

\section{Conclusions}

The findings of our pilot study suggest that patients' 


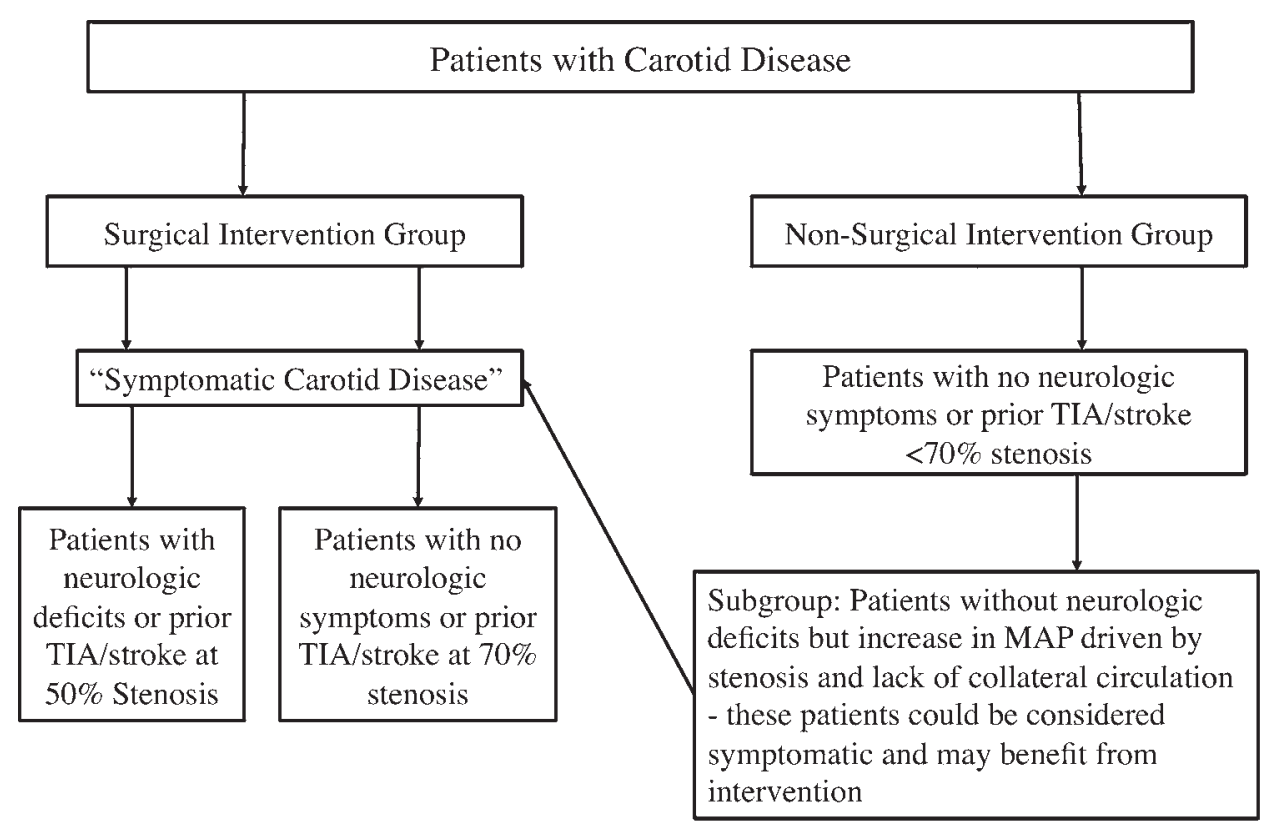

FIG. 4. Inclusion of additional subgroup of patients with absence of COW with increase in MAP $>96.3 \mathrm{~mm} \mathrm{Hg}$ driven by stenosis to current "symptomatic carotid disease" group for surgical intervention.

intrinsic intracranial collateral anatomy may contribute to their hypertensive status and influence the degree of MAP reduction following carotid artery revascularization with stenting. These results emphasize the importance of pretreatment MAP level exceeding $96.3 \mathrm{~mm} \mathrm{Hg}$ and intracranial collateral circulation imaging in patients selected for CAS.

\section{Acknowledgments}

Mr. Wali is funded through a National Institutes of Health TL1 predoctoral training grant (no. 1TL1TR001443).

\section{References}

1. Altinbas A, Algra A, Brown MM, Featherstone RL, Kappelle LJ, de Borst GJ, et al: Effects of carotid endarterectomy or stenting on blood pressure in the International Carotid Stenting Study (ICSS). Stroke 42:3491-3496, 2011

2. Bang OY, Saver JL, Kim SJ, Kim GM, Chung CS, Ovbiagele $\mathrm{B}$, et al: Collateral flow predicts response to endovascular therapy for acute ischemic stroke. Stroke 42:693-699, 2011

3. Brott TG, Halperin JL, Abbara S, Bacharach JM, Barr JD, Bush RL, et al: 2011 ASA/ACCF/AHA/AANN/AANS/ACR/ ASNR/CNS/SAIP/SCAI/SIR/SNIS/SVM/SVS guideline on the management of patients with extracranial carotid and vertebral artery disease: executive summary. J Am Coll Cardiol 57:1002-1044, 2011

4. Carretero OA, Oparil S: Essential hypertension. Part I: definition and etiology. Circulation 101:329-335, 2000

5. Chan SL, Sweet JG, Bishop N, Cipolla MJ: Pial collateral reactivity during hypertension and aging: understanding the function of collaterals for stroke therapy. Stroke 47:16181625,2016

6. Chung J, Kim BM, Paik HK, Hyun DK, Park H: Effects of carotid artery stenosis treatment on blood pressure. J Neurosurg 117:755-760, 2012

7. Chung J, Kim YB, Hong CK, Suh SH, Choi EY, Lee HJ, et al: Blood pressure-lowering effect of carotid artery stenting in patients with symptomatic carotid artery stenosis. Acta Neurochir (Wien) 156:69-75, 2014

8. Goldstein LB, Adams R, Alberts MJ, Appel LJ, Brass LM, Bushnell CD, et al: Primary prevention of ischemic stroke: a guideline from the American Heart Association/American Stroke Association Stroke Council: cosponsored by the Atherosclerotic Peripheral Vascular Disease Interdisciplinary Working Group; Cardiovascular Nursing Council; Clinical Cardiology Council; Nutrition, Physical Activity, and Metabolism Council; and the Quality of Care and Outcomes Research Interdisciplinary Working Group. Circulation 113:e873-e923, 2006

9. Heusser K, Tank J, Engeli S, Diedrich A, Menne J, Eckert S, et al: Carotid baroreceptor stimulation, sympathetic activity, baroreflex function, and blood pressure in hypertensive patients. Hypertension 55:619-626, 2010

10. Liebeskind DS: Collateral perfusion: time for novel paradigms in cerebral ischemia. Int J Stroke 7:309-310, 2012

11. Liebeskind DS, Jahan R, Nogueira RG, Zaidat OO, Saver JL: Impact of collaterals on successful revascularization in Solitaire FR with the intention for thrombectomy. Stroke 45:2036-2040, 2014

12. Lima FO, Furie KL, Silva GS, Lev MH, Camargo EC, Singhal AB, et al: The pattern of leptomeningeal collaterals on $\mathrm{CT}$ angiography is a strong predictor of long-term functional outcome in stroke patients with large vessel intracranial occlusion. Stroke 41:2316-2322, 2010

13. McKevitt FM, Sivaguru A, Venables GS, Cleveland TJ, Gaines PA, Beard JD, et al: Effect of treatment of carotid artery stenosis on blood pressure: a comparison of hemodynamic disturbances after carotid endarterectomy and endovascular treatment. Stroke 34:2576-2581, 2003

14. Oparil S, Schmieder RE: New approaches in the treatment of hypertension. Circ Res 116:1074-1095, 2015

15. Oparil S, Zaman MA, Calhoun DA: Pathogenesis of hypertension. Ann Intern Med 139:761-776, 2003

16. Osborn AG: Diagnostic Cerebral Angiography. Philadelphia: Lippincott Williams \& Wilkins, 1999 
17. Qureshi AI, Luft AR, Sharma M, Janardhan V, Lopes DK, Khan J, et al: Frequency and determinants of postprocedural hemodynamic instability after carotid angioplasty and stenting. Stroke 30:2086-2093, 1999

\section{Disclosures}

Dr. Pannell reports being a consultant for Stryker, MicroVention, and Codman.

\section{Author Contributions}

Conception and design: Khalessi, Santiago-Dieppa, Hirshman, Wali, Pannell, Olson, Cheung, Steinberg, Gupta. Acquisition of data: all authors. Analysis and interpretation of data: Khalessi, Santiago-Dieppa, Hirshman, Wali, Pannell, Cheung, Steinberg, Gupta. Drafting the article: Khalessi, Santiago-Dieppa, Hirsh- man, Wali, Pannell, Alam, Cheung, Steinberg, Gupta. Critically revising the article: all authors. Reviewed submitted version of manuscript: all authors. Approved the final version of the manuscript on behalf of all authors: Khalessi. Statistical analysis: Khalessi, Santiago-Dieppa, Hirshman, Wali, Pannell. Administrative/ technical/material support: Khalessi, Santiago-Dieppa, Hirshman, Wali, Pannell, Olson. Study supervision: Khalessi, Santiago-Dieppa, Hirshman, Wali, Pannell.

\section{Supplemental Information} Videos

Video Abstract. https://vimeo.com/206564556.

\section{Correspondence}

Alexander A. Khalessi, Department of Neurosurgery, University of California, San Diego, 200 West Arbor Dr., San Diego, CA 92103. email: akhalessi@ucsd.edu. 